\title{
Estrategias y enseñanza-aprendizaje de la lectura
}

\section{Strategies and teaching/learning to read}

\author{
Álvaro W. Santiago G. \\ Myriam C. Castillo P. \\ Dora Luz Morales*
}

\section{Resumen}

En este texto se abordan algunos de los desarrollos conceptuales que sirven de sustento teórico al proyecto "Diseño, elaboración y validación de una propuesta para la didáctica de la lectura fundamentada en metacognición", los cuales están relacionados con la noción de lectura que subyace al trabajo, las estrategias (cognitivas y metacognitivas) y su papel en la didáctica de la lectura; de igual forma, se presentan algunos aspectos relacionados con el diseño de la propuesta didáctica como tal, en lo que tiene que ver con la unidad didáctica, la planificación didáctica y los elementos que la conforman.

\section{Palabras claves:}

Lectura, comprensión, cognición, metacognición, estrategias, configuración didáctica.

\section{Abstract}

This text explores the conceptual developments useful as theoretical support to the project "Metacognitive Strategies in the Teaching and Learning of Reading", which are related to the notion that reading underlies the work, the strategies (cognitive and metacognitive) and its role in the reading didactics; moreover, some aspects showed are related to the didactic design proposal, the unit didactic, the didactic planning, and the elements that constitute it.

\section{Keywords:}

Reading, comprehension, cognition, metacognition, strategies, didactics configuration.

Artículo recibido el 6 de agosto de 2007 y aprobado el 31 de octubre de 2007

* Profesores del Departamento de Lenguas de la Universidad Pedagógica Nacional. asantiago@pedagogica.edu.co, mcastillo@pedagogica.edu.co; moradlu206@gmail.com

Grupo de Investigación en Pedagogía, Lenguaje y Comunicación, GIPELEC, clasificado en Colciencias, en la Categoría B. 


\section{Presentación}

Las consideraciones que aquí se exponen han sido resultado de las tareas que se vienen desarrollando en el marco del proyecto de investigación "Diseño, elaboración y validación de una propuesta para la didáctica de la lectura fundamentada en metacognición, para los grados $5^{\circ}$ y $6^{\circ}$ de la educación básica”, DLE-031-06, el cual forma parte del trabajo que el Departamento de Lenguas de la Universidad Pedagógica Nacional adelanta en el campo de la pedagogía del lenguaje.

El proyecto en cuestión busca diseñar y poner a prueba un programa de intervención para la enseñanza-aprendizaje de la lectura, basado en metacognición, esto es, elaborar una propuesta didáctica sustentada en la formación metacognitiva de la docente que participa en el mismo, y de un grupo de estudiantes de la educación básica primaria. Para ello, el proyecto está dividido en dos grandes fases: en la primera, se ha diseñado la propuesta didáctica y, en la segunda, esta se validará en una institución oficial de educación básica de Bogotá.

\section{La lectura}

La reflexión sobre la lectura se ha tornado en un aspecto central en distintas disciplinas (psicología, lingüística, psicolingüística, didáctica, entre otras) dado el papel fundamental que esta cumple tanto en lo individual como en lo social. A partir de las reflexiones que se han generado desde las distintas disciplinas que intentan dar cuenta de ella, la lectura ha dejado de concebirse como el mero reconocimiento de unos signos gráficos, o la simple transcripción de lo gráfico a lo verbal, o la decodificación de unos símbolos alfabéticos, para convertirse, antes que nada, en un proceso dinámico, en un trabajo de carácter cognitivo, mediante el cual un individuo adelanta una serie de operaciones mentales encaminadas a reconstruir el significado de un texto, pues como plantean De Vega et ál. "el proceso lector completo consiste en la construcción del significado global del texto" (1990, p. 17).

Pero esta actividad no se centra únicamente en la apropiación del contenido sino que, a su vez, se constituye en un proceso de construcción y producción de aquél, puesto que es el lector quien activa el proceso semiósico interpretativo ${ }^{1}$ (Santiago, Castillo y Ruiz, 2005); en consecuencia, la lectura se entiende como una actividad de comprensión y producción de sentido; no es un simple trabajo de decodificación sino un proceso de interrogación, participación y actualización por parte de un receptor activo que la reconoce como un proceso de cooperación textual.

Esta búsqueda y construcción de significado implica que el lector efectúe una serie de operaciones cognitivas (abstracción, análisis, síntesis, inferencia, predicción, comparación) en las que pone en juego sus conocimientos, intereses y estrategias, con los aspectos que proporciona el texto, en unas circunstancias determinadas. De esta forma, la lectura se torna en una interacción entre el lector, texto y contexto.

Según este último planteamiento, se asume un modelo interactivo de la lectura, en el cual el lector, ubicado en un contexto particular, pone en diálogo sus conocimientos e intereses con los contenidos y propósito(s) que el texto posee, los cuales se manifiestan a través de indicios lingüísticos, semióticos y retóricos; diálogo mediado por los procesos cognitivos que el lector pone en marcha. Esta concepción interactiva surge como alternativa frente a los modelos ascendente y descendente ${ }^{2}$.

1 Siguiendo a Bobes (1989, p. 122-124), el proceso semiósico le brinda al individuo la posibilidad de generar significado; dicho proceso supone la presencia de tres elementos básicos: el emisor (locutor), el signo y el receptor (interlocutor). Este proceso, según la presencia de los elementos y el papel que desempeñen, puede presentar estas variantes: proceso de expresión, de significación, de comunicación, de interacción y de interpretación.

2 Como complemento a esto, Cassany (2006) plantea que hay tres concepciones sobre la lectura: la lingüística, la psicolingüística y la sociocultural. La concepción lingüística propone que el significado se encuentra en el texto, así, "leer es recuperar el valor semántico de cada palabra y relacionarlo con el de las palabras anteriores y posteriores. El contenido surge de la suma del significado de todos los vocablos y oraciones" (Cassany, 2006, p. 25). La concepción psicolingüística plantea que el significado no está sólo en el texto sino también en la mente del lector, de tal forma que "leer no sólo exige conocer las unidades y las reglas combinatorias del idioma. También requiere desarrollar las habilidades cognitivas implicadas en el acto de comprender: aportar conocimiento previo, hacer inferencias, formular hipótesis y saberlas reformular, etc." (p. 32). Finalmente, la concepción sociocultural plantea que "leer no es sólo un proceso psicobiológico realizado con unidades lingüísticas y 
El modelo de procesamiento ascendente (bottom up) propone que la lectura implica ir de la identificación e integración de los niveles inferiores (grafías, palabras, frases) hasta llegar a las unidades lingüísticas superiores (oraciones, texto); en este modelo, la comprensión se entiende entonces como reconocimiento jerárquico de unidades lingüísticas, esto es, un proceso de decodificación. Por su parte, el modelo descendente (top down) sostiene que el proceso lector va de la mente del individuo al texto, de tal forma que éste se erige como el protagonista del proceso, dado que se plantea que los conocimientos que el lector posea sobre el mundo en general y sobre el tema del texto en particular, le permiten entenderlo de forma más fácil (Castelló, 1997, p. 187). En este orden de ideas, Smith (1990) argumenta que para leer, un individuo necesita tanto de la información visual, que ofrece el texto, como de la información no visual, que corresponde a los conocimientos de diversa índole que tiene en su cerebro, bajo la forma de una teoría del mundo. Según este autor, la relación entre estos dos tipos de informaciones necesarias para leer es recíproca, de tal forma que entre más información no visual posea el lector, requerirá de menor información visual, y entre menos información no visual posea, precisará de mayor información visual.

Desde la perspectiva del modelo interactivo, se asume entonces la comprensión lectora como un proceso constructivo en el que se "requiere el esfuerzo deliberado del lector para ir interpretando el significado de un texto" (Castelló, 1997); en este sentido, la lectura es un proceso dialéctico que se establece entre el sujeto lector y el texto, proceso que implica una negociación "entre lo que el lector va interpretando y las características particulares del texto" (p. 188), así como las condiciones mismas en las que se lleva a cabo el ejercicio lector. Según esto, cada uno de estos componentes (sujeto, texto y

capacidades cognitivas. También es una práctica cultural insertada en una comunidad particular, que posee una historia, una tradición, unos hábitos y unas prácticas comunicativas especiales" (p. 38), de forma que al leer se hace necesario recurrir a los aspectos cognitivos pero también a todo el conocimiento sociocultural para poder comprender el texto. contexto) aporta una serie de elementos al proceso de comprensión lectora.

El texto ofrece informaciones de tipo lingüístico, textual y discursivo (Tardif, 1997). Las informaciones de tipo lingüístico tienen como límite el nivel de la oración, es decir, se presentan a nivel intraoracional; estas informaciones corresponden al sistema formal de la lengua (gramática). Las informaciones de tipo textual están referidas a las relaciones interoracionales, esto es, la manera como se integran las oraciones en el nivel textual (coherencia, cohesión). Las informaciones de orden discursivo (pragmático) "tienen que ver con la situación comunicativa, el tipo de discurso y las relaciones entre el texto y el contexto" (Tardif, 1997, p. 183).

El contexto, entre tanto, se relaciona con los elementos del entorno en el cual se lleva a cabo el proceso lector; así, el contexto corresponde "a todas aquellas situaciones que rodean y propician la interacción entre el texto y el lector, de tal manera que aparecen multiplicidad de factores alrededor del acto lector: propósitos del lector, tipo de texto, condiciones de lectura, situación específica, entre otros" (Santiago, Castillo y Ruiz, 2005, p. 23).

El lector aporta todos sus conocimientos, procesos mentales e intereses. Siguiendo a Tardif, el lector se constituye en elemento esencial dado que es él quien posibilita la reconstrucción del significado del texto, el cual se recrea a partir de la interacción de sus conocimientos previos 3 , de diverso tipo, con las informaciones que ofrece el texto y el contexto.

En consecuencia, teóricamente asumimos un modelo interactivo de la lectura; desde esta perspectiva, aquélla se concibe como una actividad cognitiva compleja, en la que el lector se constituye

3 De acuerdo con Goodman (1996), la comprensión lectora es un proceso a lo largo del cual el lector va construyendo el significado del texto. Para ello, éste se vale de dos tipos de conocimientos: a) los conocimientos previos conceptuales que corresponden al saber enciclopédico o conocimiento del mundo que posee el individuo, conocimiento que se expresa en términos de esquemas mentales, por ejemplo; y b) los conocimientos previos letrados, es decir, los conocimientos que el individuo posee sobre el código escrito en particular, agrupados en conocimientos sobre aspectos materiales o paratextuales (material de soporte, formatos, tipografía, diseño gráfico) así como aspectos textuales (alfabéticos, morfosintácticos, léxicos, semánticos y pragmáticos). 
en un procesador de la información que le ofrece el texto. En este punto, atendiendo los planteamientos de Tardif en relación con el saber-leer, la interacción texto-lector está mediada por los conocimientos de éste, que se pueden agrupar en conocimientos declarativos, procedimentales y condicionales.

Los conocimientos declarativos (el qué) están asociados a conocimientos sobre el mundo en general, la lengua y, desde luego, la escritura. Entre tanto, los conocimientos procedimentales tienen que ver con el cómo adelantar el proceso lector y están relacionados con los procesos y estrategias de orden cognitivo que le permiten a la persona que pueda desarrollar una tarea determinada; en lectura, es a través de las estrategias cognitivas que un lector puede poner en relación diferentes informaciones provenientes de niveles diversos. Es a través de estrategias cognitivas como establece lazo entre sus conocimientos anteriores y las informaciones suministradas por el texto para su comprensión" (Tardif, 1997, p. 187).

Forman parte de estos procesos mentales aspectos como abstraer, analizar, comparar, clasificar, organizar, identificar, inferir, recordar, elaborar, que se constituyen en aspectos susceptibles de mejorar y de abordar como contenidos escolares, con el fin de potenciar el desempeño lector de los educandos.

Por su parte, los conocimientos condicionales también tienen que ver con la acción y están asociados al cuándo y al porqué adelantar una tarea; "en lectura aseguran que el alumno recurra a la estrategia adecuada en el momento justo" (Tardif, 1997, p. 187), de tal forma que le permiten al lector seleccionar las estrategias, los recursos y los procedimientos necesarios para realizar la lectura; de otro lado, le permiten justificar el porqué de su actuación. Además, los conocimientos condicionales incluyen las estrategias metacognitivas que le ofrecen al individuo la posibilidad de controlar la realización de la tarea, en la medida en que le posibilitan analizarla, definir objetivos, establecer estrategias, supervisar el proceso y evaluar el resultado.

Saber-leer se concibe como una actividad compleja que supone la utilización de diversos tipos de conocimientos que se ponen al servicio de la com- prensión e interpretación de un texto. Desde esta óptica, la enseñanza de la lectura implica abordar, además de contenidos conceptuales y procedimientales, conocimientos de orden metacognitivo, esto es, posibilitar en los lectores en formación el desarrollo de estrategias que les brinden la opción de controlar y evaluar su proceso lector.

Lo anterior lleva a analizar los tipos de contenidos que se deben tener presentes al momento de enseñar a comprender textos. Según Rincón et ál. (2003), el papel del docente en el proceso de enseñanza de la comprensión textual apunta a ofrecer al estudiante el andamiaje necesario que lo lleve a ser lector autónomo 4 . Para ello, se presentan dos tendencias con respecto a los contenidos de la enseñanza de la comprensión:

a) Enseñar estrategias de comprensión que involucran lo cognitivo y lo metacognitivo. Estas estrategias se asocian a las tres fases que, desde la óptica de Solé (1998), involucra el ejercicio lector: prelectura o fase de anticipación en la que se encuentra, definir objetivos, indagar sobre conocimientos previos, formular predicciones, plantear interrogantes con respecto al texto; durante la lectura o fase de construcción, que supone aspectos como centrarse en el contenido principal, controlar la comprensión, identificar afirmaciones, formular hipótesis y evaluarlas, formular preguntas y responderlas, buscar ayuda en caso de tener dificultades de comprensión; y después de la lectura o fase de evaluación, que contempla dar cuenta del proceso por medio de diversos recursos: resúmenes, mapas conceptuales, mapas mentales, cuadros sinópticos, reseñas, entre otros.

El planteamiento que subyace a esta tendencia

"Propone que mediante la creación de situaciones significativas y globales de lectura y, en un proceso por etapas, se vaya logrando un traspaso desde la responsabilidad del maestro en la interpretación

4 De acuerdo con Smith, "la responsabilidad del maestro no radica en enseñar a los niños a leer sino en posibilitarles que aprendan a leer" (1990, p. 22). 
de lo que se lee, hasta la autonomía completa del alumno" (Rincón et al, 2003, p. 101).

Esto quiere decir que la propuesta didáctica contempla la transferencia gradual del proceso de aprendizaje del docente al estudiante, de forma que en los primeros momentos el profesor es el responsable del control de la actividad de aprendizaje, asumiendo el papel de guía y modelo de la actividad cognitiva y metacognitiva, y poco a poco va llevando al estudiante para que éste tome por su cuenta el control del proceso. De acuerdo con Mateos (2001), los métodos que permiten adelantar esta transferencia del proceso de aprendizaje son: la instrucción explícita, la práctica guiada, la práctica cooperativa y la práctica individual. Gracias a estos métodos, "la instrucción transcurre de este modo a lo largo de un continuo de responsabilidad en la realización de la tarea que se va desplazando progresivamente del profesor hacia el alumno" (Mateos, 2001, p. 103).

b) Enseñar los usos sociales de la lectura. Al respecto, Lerner (2001) argumenta que si el propósito educativo en la enseñanza de la lectura y de la escritura apunta a formar a los estudiantes como ciudadanos de la cultura escrita, entonces, "el objeto de enseñanza debe definirse tomando como referencia fundamental las prácticas sociales de lectura y escritura" (Lerner, 2001, p. 85); esto significa tener en cuenta los propósitos de la lectura según la situación, las maneras de leer, las relaciones del lector con otros lectores y con los textos, en fin, supone "concebir como contenidos fundamentales de la enseñanza los quehaceres del lector, los quehaceres del escritor" (p. 96). En otras palabras, se asumen como contenidos escolares todas las acciones que ejecutan los lectores y escritores cuando enfrentan las tareas lectora y escritora. Siguiendo a Lerner, entre las cosas que hace un lector y que suponen interacción con otros, se encuentran "comentar o recomendar lo que se ha leído, compartir la lectura, confrontar con otros lectores las interpretaciones generadas por un libro o una noticia, discutir sobre las intenciones implícitas en los titulares de cierto periódico..." (p. 96); otros quehaceres son de carácter individual: anticipar, releer un fragmento, dejar de lado aspectos que no interesan, valorar el texto, definir objetivos, entre otros.

Así, un uso de la lectura apunta a conseguir información o aprender, para lo cual los lectores emplean una serie de recursos cognitivos (identificar, seleccionar, abstraer, generalizar, comparar, inferir, predecir...) y metacognitivos (definir un objetivo, determinar estrategias para adelantar la tarea, supervisarla, autorregularla y evaluarla), que se hacen evidentes en actividades tan sencillas y cotidianas como formular preguntas, establecer predicciones, subrayar, tomar notas, identificar afirmaciones, elaborar resúmenes, etc., aspectos que se constituyen en los indicios (indicadores de logro, si se quiere) que permiten evidenciar tanto el proceso como el resultado del ejercicio lector y que, como se ha señalado, se constituyen en parte de los contenidos que se deben abordar en la enseñanzaaprendizaje de la lectura. Según esto, se plantea que dichos contenidos -estrategias y usos sociales- no son excluyentes sino complementarios para formar lectores competentes, es decir, individuos que logren apropiarse de la lectura y se conviertan en ciudadanos de la cultura escrita.

\section{Las estrategias y la enseñanza-aprendizaje de la lectura}

Según lo desarrollado, el saber leer supone, desde la perspectiva del sujeto lector, la activación de conocimientos declarativos, procedimentales y condicionales; ahora bien, el proceso de enseñanzaaprendizaje de la lectura, desde la óptica del docente, implica decidir de estos conocimientos cuáles va a privilegiar y, en consecuencia, qué tipos de contenidos va a trabajar en el aula. A partir de lo expuesto, hay planteamientos que asumen como contenidos escolares en la enseñanza de la lectura las estrategias (cognitivas y metacognitivas); esto implica conocer los aspectos relacionados con los procesos mentales que conlleva el aprender -dimensión cognitiva ${ }^{5}$ - y

5 El concepto cognición está referido al conocimiento que el individuo tiene del mundo, el cual se encuentra en la memoria de los 
cómo controlarlos - dimensión metacognitiva ${ }^{6}$, de tal forma que, como plantean Gaskins y Thorne, hay dos categorías de estrategias: cognitivas y metacognitivas. "Las cognitivas ayudan a los estudiantes a lograr las metas de su empresa cognitiva, las estrategias metacognitivas les ofrecen información sobre el avance hacia sus metas" (Gaskins y Thorne, 1999, pp. 87-88). Al hablar de enseñanza-aprendizaje de estrategias se deben tener en cuenta las dos categorías.

De acuerdo con los planteamientos de Gaskins y Thorne, el trabajo con estrategias en el aula de clase se sustenta en el hecho de que las investigaciones han brindado la posibilidad de establecer que uno de los aspectos que permite caracterizar a los estudiantes que logran buenos resultados académicos, con respecto a aquellos que presentan problemas en su desempeño escolar, tiene que ver con el uso de estrategias, de forma que

"los buenos alumnos [...] son conscientes de factores que afectan el aprendizaje y de cómo poner en marcha un conjunto de estrategias. También controlan factores que afectan la enseñanza y el pensamiento, manejando activamente las estrategias que son necesarias para tener éxito" (Gaskins y Thorne, p. 49).

Para efectos de diseñar la propuesta para la enseñanza-aprendizaje de la lectura con una base metacognitiva, uno de los objetivos del proyecto DLE-031-06 que se presentó al inicio de este documento, el equipo de investigación se ha basado en la propuesta de Gaskins y Thorne, sustentada en el trabajo que estas investigadoras han desa-

individuos "a través de diferentes sistemas, códigos o formatos de representación y es adquirido, manipulado y utilizado para diferentes fines por el entero sistema cognitivo" (Mayor, Suengas y González, 1993, p. 13).

6 La metacognición se puede entender como "la cognición sobre la cognición, el conocimiento sobre el conocimiento, el pensamiento sobre el pensamiento" (Mayor, Suengas y González, p. 51); esto está relacionado con la conciencia que se tiene del conocimiento y cómo se obtiene. En este orden de ideas, la metacognición se caracteriza como un conocimiento autorreflexivo que se logra a través de la autoobservación; ésta tiene por objeto el conocimiento de los mecanismos implicados o responsables del conocimiento y de las operaciones mentales responsables del mismo; de esta forma, se puede entender como un tipo de conocimiento que permite controlar el aprendizaje y la misma actividad cognitiva (Mateos, 2001). rrollado en la escuela Benchmark, en la que han planteado un currículo que incluye como parte de los contenidos el trabajo con estrategias cognitivas y metacognitivas.

Según estas autoras, las estrategias se constituyen en elementos claves para aprender, pensar y resolver problemas. Desde esta perspectiva teórica, las estrategias se pueden caracterizar como las acciones y pensamientos de los estudiantes que emergen durante el proceso de aprendizaje; en este sentido, se tornan en recursos orientados hacia metas, recursos que facilitan el desempeño y la obtención de la meta propuesta, de tal forma que implican una secuencia de actividades que permiten obtener un fin7. Ahora bien, estas acciones o secuencia de actividades pueden ser específicas para una tarea particular o generales, es decir, válidas para cualquier tarea 8 ; finalmente, como ya se indicó, las estrategias se ubican en dos categorías: cognitivas, que ayudan a conseguir las metas (hacer avances), y metacognitivas, que ofrecen información sobre el avance del proceso (controlar los avances).

Las estrategias cognitivas están asociadas a los procesos mentales que supone la realización de una actividad cognitiva, estos son: centrar la atención, recoger información, ensayar, recordar, analizar, elaborar-generar, organizar-integrar, evaluar, monitorear. Por su parte, las estrategias metacognitivas están relacionadas con el control de estos procesos mentales, de forma que éstas tienen que ver con planificar, supervisar y evaluar la tarea. Con respecto a las estrategias cognitivas asociadas directamente

7 Para Monereo, la estrategia se puede entender como el "conjunto de acciones que se realizan para obtener un objetivo de aprendizaje" (Monereo, 2002, p. 24). Por su parte, López y Arciniegas entienden por estrategia "la secuencia de actividades intencionales y deliberadas en las cuales se involucra constantemente el individuo para lograr las metas que se ha propuesto" (López y Arciniegas, 2004, p. 43).

8 De acuerdo con el trabajo de Gaskins y Thorne, pensar supone poner en acción procesos mentales tales como centrar la atención, recoger información y recordar; éstos son procesos generales y se asocian con estrategias generales, es decir, válidas para realizar cualquier tarea. Estos procesos generales llevan a establecer procesos específicos, particulares según la tarea, los cuales se relacionan con estrategias como comparar, resumir, clasificar, interpretar, criticar, buscar presupuestos, formar imágenes, organizar datos, recordar, formular hipótesis, etc. 
al proceso de producción de sentido, en el cual se ubica la lectura, Gaskins y Thorne (1999, pp. 98-100) formulan: explorar, acceder al conocimiento previo, predecir, formular hipótesis y/o plantear objetivos, comparar, hacer inferencias, generar preguntas $y$ pedir aclaraciones, seleccionar ideas importantes, parafrasear o resumir, organizar ideas clave, entre otras. Según estas consideraciones, el equipo de investigación ha definido cuatro procesos mentales básicos, a los cuales se le asocian estrategias cognitivas y metacognitivas. Estos procesos mentales son: centrar la atención, analizar, organizar y elaborar.

Como se planteó líneas atrás, las estrategias en lectura se pueden ubicar en tres grandes momentos del proceso: antes, durante y después. Así, al relacionar los procesos mentales con estas estrategias se puede establecer que centrar la atención es un proceso asociado con estrategias de prelectura, pues aquí se ponen en marcha acciones como formular objetivos, explorar, plantear hipótesis, activar conocimientos previos; por su parte, analizar tiene que ver con el desarrollo del proceso lector dado que, entre otros aspectos, supone identificar temas e ideas principales; entre tanto, organizar y elaborar se asocian con las estrategias de poslectura puesto que organizar implica formalizar la comprensión a través del uso de distintos recursos gráficos y esquemáticos; y elaborar comporta resumir o parafrasear. Ahora bien, las estrategias metacognitivas para cada uno de estos procesos tienen que ver con planificar la tarea, supervisar su ejecución y evaluar el proceso y el resultado. Lo anterior lo podemos representar así:

\begin{tabular}{|c|c|c|c|c|}
\hline $\begin{array}{l}\text { Proceso } \\
\text { mental }\end{array}$ & Actividades cognitivas & $\begin{array}{l}\text { Fase proceso } \\
\text { lector }\end{array}$ & $\begin{array}{l}\text { Estrategias } \\
\text { metacognitivas }\end{array}$ & $\begin{array}{l}\text { Actividades } \\
\text { metacognitivas }\end{array}$ \\
\hline \multirow{4}{*}{$\begin{array}{l}\text { Centrar la } \\
\text { atención }\end{array}$} & Formular objetivos & & \multirow{4}{*}{ Planificar } & \multirow{8}{*}{$\begin{array}{l}\text { Analizar la tarea (propósito, } \\
\text { extensión grado de dificultad). } \\
\text { Planificar la ejecución de la tarea } \\
\text { (definición de tiempo de ejecución, } \\
\text { de estrategias). } \\
\text { Determinar los conocimientos que } \\
\text { se poseen con respecto a la tarea. } \\
\text { Identificar puntos clave de la tarea. } \\
\text { Tomar notas, subrayar, hacer } \\
\text { preguntas, releer. } \\
\text { Elaborar resúmenes y esquemas } \\
\text { que den cuenta del texto. } \\
\text { Analizar errores cometidos y } \\
\text { plantear soluciones. }\end{array}$} \\
\hline & Explorar & & & \\
\hline & Formular hipótesis & - Prelectura & & \\
\hline & Activar conocimientos previos & & & \\
\hline \multirow{2}{*}{ Analizar } & Identificar temas & & \multirow[b]{3}{*}{ Supervisar } & \\
\hline & Identificar ideas principales & - Durante la lectura & & \\
\hline Organizar & Formalizar la comprensión & & & \\
\hline Elaborar & Resumir/parafrasear & Poslectura & Evaluar & \\
\hline
\end{tabular}

\section{Hacia una propuesta de configuración didáctica}

A partir de lo expuesto, el equipo de investigación ha diseñado la propuesta didáctica. Para ello, se han tenido en cuenta las nociones de configuración didáctica, secuencia didáctica, unidad didáctica y planificación didáctica, con el fin de determinar la manera como se presentará la propuesta, objeto del proyecto.

La configuración didáctica tiene que ver con la manera particular que emplea el docente para favorecer y facilitar el proceso de aprendizaje de sus estudiantes; desde la perspectiva de Litwin (1997), la configuración didáctica es un modo particular de práctica docente que implica una forma peculiar de organizar y desarrollar la enseñanza, situada en un contexto específico, de forma que se constituye en "la manera particular que despliega el docente para favorecer los procesos de construcción del conocimiento" (Litwin, p. 97).

La secuencia didáctica hace referencia, según Camps (2003), a la estructura de acciones vinculadas entre sí, de naturaleza intencional, que se organizan para alcanzar algún aprendizaje. Según esto, la secuencia implica la organización de acciones que tienen como finalidad propiciar el aprendizaje, teniendo en cuenta las características mismas de la interacción que se da entre estudiante y profesor, los 
tipos de discursos que circulan en el contexto educativo y todos los materiales de soporte que permiten el desarrollo de las acciones educativas en sí.

La unidad didáctica se entiende como una forma de planificar el proceso de enseñanza-aprendizaje alrededor de un elemento de contenido, en este caso, las estrategias como contenidos escolares. Este elemento se constituye en el eje que permite integrar los distintos elementos que intervienen en el proceso educativo, asociados al docente, el estudiante, los contenidos, los recursos, las intenciones que orientan el proceso, entre otros, a la vez que posibilita el desarrollo de éste en unas condiciones contextuales particulares.

Según lo anterior, la configuración didáctica, relacionada con las maneras de hacer, supone tipos, entre los cuales se pueden encontrar los proyectos de aula, las secuencias didácticas y las unidades didácticas. La propuesta que se plantea en el marco del proyecto toma -como modelo de formulaciónla unidad didáctica, de forma que se han definido cuatro unidades, ordenadas de forma secuencial: centrar la atención, analizar, organizar y elaborar, según los procesos mentales establecidos.

La noción de unidad didáctica conlleva la de planificación didáctica. La planificación se debe entender, fundamentalmente, como una manera de organizar el trabajo en el aula, como una hipótesis de trabajo, en consecuencia, flexible, falible y perfectible. De acuerdo con Lomas (1999), la planificación didáctica debe servir de guía para organizar la práctica educativa, ello debido a que contempla actividades de enseñanza-aprendizaje, tiempos de ejecución, metodologías, finalidades, contenidos, recursos, espacios y formas de evaluación. Esta planificación se caracteriza, siguiendo a Lomas, por ser adecuada al entorno sociocultural, útil, flexible y posible; tiene como funciones organizar el proceso de enseñanza-aprendizaje en el aula, asegurar la coherencia entre los objetivos educativos y la práctica, fomentar la reflexión sobre la práctica docente, facilitar el aprendizaje de los educandos y tener en cuenta la diversidad de intereses, actitudes y aptitudes de los estudiantes.
Desde la perspectiva de Gvirtz y Palamidessi, una planificación didáctica debe tener en cuenta una serie de variables o aspectos de la realidad que el docente debe considerar al momento de "planificar y desarrollar una actividad sistemática de enseñanza" (Gvirtz y Palamidessi, p. 188). Así, al adelantar el diseño de una planificación didáctica se hace necesario contemplar una serie de variables o elementos que orientan y, en cierta medida, determinan su configuración. Tales variables están relacionadas con la definición de metas; objetivos, logros; la selección y secuenciación de los contenidos; la determinación de las orientaciones metodológicas; la elección de recursos y materiales; el establecimiento de la forma de evaluación. Según este último aspecto, en las planificaciones que el equipo ha diseñado en el marco de la propuesta didáctica se han contemplado estos elementos:

Objetivos: entendidos como las finalidades generales que se persiguen con la unidad didáctica, esto es, los propósitos que, desde la óptica del profesor, orientan el proceso de enseñanza-aprendizaje.

Logros: relacionados con la definición del perfil del estudiante que se espera al final del proceso de enseñanza-aprendizaje; en este sentido, el logro tiene que ver con los adelantos (conceptuales, procedimentales, condicionales y actitudinales) que el estudiante debe adelantar a lo largo del proceso formativo, lo cual se hace evidente en una serie de desempeños.

Indicadores de logro: el conjunto de señales o indicios que permiten evidenciar el nivel de logro en que se encuentra el estudiante; de otro lado, los indicadores se constituyen en los pasos que el estudiante debe dar para llegar al logro, de esta forma se tornan en aspectos que guían la intervención educativa y determinan tipos de actividades, así como aspectos relacionados con metodología, recursos y evaluación.

Contenidos: vistos como el conjunto de saberes o formas culturales de diversa índole (conceptos, da- 
tos, hechos, procedimientos, estrategias, principios, valores, actitudes, normas, etc.) que se consideran adecuados para posibilitar el proceso de aprendizaje-enseñanza. En consecuencia, los contenidos hacen referencia a "las informaciones, objetos o instrumentos que la sociedad considera de utilidad para promover el desarrollo social y personal de sus ciudadanos" (Gallego y Salvador, 2002, p. 132). Los contenidos se pueden clasificar en conceptuales o declarativos (hechos, conceptos, datos, principios, leyes), procedimentales (destrezas y habilidades de uso) y actitudinales (valores, normas, actitudes).

Metodología o definición de las actividades que han de adelantar tanto estudiantes como profesor, mediante las cuales se ponen en juego prácticas de enseñanza-aprendizaje; en este sentido, la metodología tiene que ver con las estrategias que se emplean para adelantar la interacción profesor-estudiante, profesor-estudiantes, estudiantes-estudiantes y estudiantes-contenidos. En este aspecto se puede hacer mención de la instrucción explícita, el modelado metacognitivo, la práctica guiada, el trabajo cooperativo y la práctica autónoma.
Recursos o elementos que "el profesor prevea emplear en el diseño o desarrollo del currículo (por su parte o la de los alumnos) para aproximar o facilitar los contenidos, mediar las experiencias de aprendizaje, provocar encuentros o situaciones, desarrollar habilidades cognitivas, apoyar sus estrategias metodológicas, o facilitar o enriquecer la evaluación" (Blázquez y Lucero, 2002, p. 186).

Evaluación: instrumento que busca dar cuenta del proceso formativo que se ha adelantado y, así, establecer el grado de desarrollo de los educandos, a la vez que permite identificar problemáticas tanto de los estudiantes como de la propuesta en sí; de esta forma, la evaluación se dirige hacia los estudiantes, en quienes se busca fomentar la autoevaluación, aspecto clave en la metacognición, a la vez que contempla la valoración de la propuesta didáctica, para determinar los alcances, aciertos, dificultades y falencias que ésta pueda presentar.

Para ilustrar lo que se ha descrito, a continuación se presenta la propuesta de unidad didáctica para la estrategia cognitiva centrar la atención:

\section{UNIDAD DIDÁCTICA I}

1. Tema: Estrategias metacognitivas de comprensión lectora asociadas al proceso cognitivo centrar atención.

2. Duración: ocho sesiones de dos horas (16 h).

3. Objetivo: conocer y utilizar estrategias metacognitivas de prelectura asociadas al proceso cognitivo centrar la atención: formular objetivos, explorar, plantear hipótesis y activar conocimientos previos.

4. Logros

4.1 Planifica, supervisa y evalúa la formulación de objetivos en relación con la tarea propuesta. Indicadores de logro:

- Analiza la tarea.

- Comprende la tarea.

- Plantea la finalidad de la tarea y establece objetivos para el desarrollo de ésta.

- Autorregula el proceso de formulación de objetivos.

4.2 Planifica, supervisa y evalúa la exploración del texto y la formulación de hipótesis en relación con éste.

Indicadores de logro:

- Revisa el texto que va a leer: extensión, tamaño de la letra, apartados...

- Analiza el título.

- Identifica partes del texto (subtítulos, párrafos...).

- Propone hipótesis sobre el posible tema.

- Autorregula el proceso de exploración y formulación de hipótesis. 
4.3 Planifica, supervisa y evalúa la activación de conocimientos previos sobre el tema que aborda el texto.

Indicadores de logro:

- Formula preguntas sobre el tema del texto.

- Identifica aspectos relacionados con el tema del texto.

- Hace uso de estrategias para activar conocimientos previos.

- Representa los conocimientos previos de forma gráfica.

- Autorregula el proceso de activación de conocimientos previos.

5. Contenidos

5.1 Declarativos:

- La lectura, tipos de lectura...

- Tipos de textos: narrativos, descriptivos, expositivos, argumentativos.

- Estrategias cognitivas y metacognitivas.

5.2 Procedimentales:

- Estrategias cognitivas: centrar atención.

- Estrategias metacognitivas: formulación de objetivos, formulación de hipótesis, activación de conocimientos, manejo de tablas KLW.

5.3 Condicionales:

- Planificación.

- Supervisión.

- Evaluación.

6. Metodología

6.1 Profesor:

- Presentación de los contenidos: estrategias.

- Presentación de los objetivos y de los logros.

- Instrucción explícita: ejercicio de modelado metacognitivo para cada estrategia y logro.

6.2 Profesor-estudiantes:

- Práctica guiada: aplicación orientada por el profesor (ofrecer pistas y hacer preguntas).

- Desarrollo de interrogación metacognitiva.

6.3 Estudiantes

- Trabajo cooperativo: ejercicios de aplicación entre pares.

- Trabajo individual: transferencia de lo aprendido a otras situaciones.

- Desarrollo y práctica de la autointerrogación metacognitiva.

6.4 Profesor-estudiantes

- Análisis y discusión metacognitiva:

- Analizar la efectividad de la estrategia.

- Comprender los procesos cognitivos y metacognitivos implicados en el uso de la estrategia.

- Diferenciar las fases más importantes y menos relevantes en la utilización de la estrategia.

- Valorar diferentes maneras de utilizar o adaptar la estrategia.

- Formalización de aprendizajes.

7. Recursos

- Textos expositivos, tablero, portafolio, fotocopias, papel, lápiz, colores, libros, formatos de evaluación, talleres.

8 Evaluación

8.1 Evaluación a estudiantes:

- Evaluación del proceso.

- Evaluación de los aprendizajes.

- Autevaluación.

8.2 Evaluación del trabajo:

- Metodología.

- Proceso.

- Resultado. 
Tal como se planteó, la planificación contempla la formulación de un propósito o intención formativa del profesor que busca dar a conocer y propiciar el uso de estrategias metacognitivas relacionadas con el proceso centrar la atención; este objetivo lleva a la formulación de unos logros, enfocados en el estudiante, que buscan desarrollar su competencia lectora. Ligados a éstos, se establecen los indicadores que permitan llegar al logro propuesto. A partir de ellos se establecen los contenidos declarativos, procedimientales y condicionales, de los que priman los dos últimos, esto es, las estrategias cognitivas y metacognitivas asumidas como contenidos escolares en la enseñanza-aprendizaje de la lectura. Como se observa, la intervención pretende, a través de métodos como la instrucción explícita, la práctica guiada, la práctica cooperativa y la practica individual, ceder de manera gradual al estudiante la supervisión, el control y la evaluación del proceso y distanciar de ellas progresivamente al profesor, para así formar un lector intencional y autónomo. Para efectos de adelantar el trabajo, se han emplado textos expositivos como recurso básico para llevar a cabo la labor, asimismo, teniendo en cuenta los textos, se han elaborado talleres para el trabajo guiado, cooperativo y autónomo en los que se aborda la formulación de objetivos e hipótesis, la activación de conocimientos previos, así como la reflexión metacognitiva. Finalmente, se contempla la evaluación como un aspecto permanente que pueda dar cuenta del proceso y del resultado, utilizando para ello la

\section{Bibliografía}

Blázquez, Florentino y Lucero, Manuel. (2002). Los medios y recursos en el proceso didáctico. En: Medina, Antonio y Salvador Francisco (coords.). Didáctica general. Madrid: Pearson Educación.

Bobes, María. (1989). La semiología. Madrid: Síntesis, 1989.

Burón, Javier. (1996). Enseñar a aprender: Introducción a la metacognición. Bilbao: Mensajero.

Camps, Anna. (2003). Secuencias didácticas para aprender a escribir. Barcelona: Graó. lista de control, la escala de valoración descriptiva por logros e indicadores, las fichas de autoevaluación y los portafolios.

En síntesis, en el marco del proyecto se ha asumido como perspectiva teórica la lectura como un proceso complejo de reconstrucción de significado, que implica la interacción de lector, texto y contexto; interacción mediada por los conocimientos previos del lector: declarativos, procedimentales y condicionales. Desde esta perspectiva, la didáctica de la lectura implica abordar, como parte de los contenidos escolares, no sólo conocimientos declarativos y procedimentales sino aspectos metacognitivos, además de los usos sociales de la lectura, con el fin de que los estudiantes puedan conocer y desarrollar estrategias adecuadas para así controlar y evaluar de forma autónoma su proceso lector. En aras de dar cuenta de este objetivo, se ha definido, en términos de configuración didáctica, la unidad didáctica como una forma de planificar el proceso de enseñanza-aprendizaje que tiene como eje de contenido las estrategias cognitivas y metacognitivas. Dicha unidad didáctica involucra la noción de planificación, entendida como hipótesis que orienta el trabajo pedagógico en la medida en que contempla objetivos, logros, indicadores de logro, definición de contenidos, estrategias metodológicas, recursos y materiales, y formas de evaluación; con base en estos elementos, se diseñan entonces las unidades que conforman la propuesta didáctica para la enseñanza-aprendizaje de la lectura. صి
Cassany, Daniel. (2006). Entre líneas: Sobre la lectura contemporánea. Barcelona: Anagrama.

Castelló, Monserrat. (1997). Las estrategias de aprendizaje en el área de la lectura. En: Monereo, Carles. (coord.). Estrategias de aprendizaje. Madrid: Antonio Machado Libros.

De Vega, Manuel et ál. (1990). Lectura y comprensión: una perspectiva cognitiva. Madrid: Alianza.

Gallego, José y Salvador, Francisco. (2002). Los contenidos en el proceso didáctico. En: Medina, Antonio y salva- 
dor Francisco (coords.). Didáctica general. Madrid: Pearson Educación.

Gaskins, Irene y Thorne, Elliot. (1999). Cómo enseñar estrategias cognitivas en la escuela: El manual Benchmark para docentes. Barcelona: Paidós.

Goodman, Kenneth. (1996). “La lectura, la escritura y los textos escritos: una perspectiva transaccional sociopsicolingüística". En: Textos en contexto 2: Los procesos de lectura y escritura. Buenos Aires: Lectura y vida.

Gvirtz, Silvina y Palamidessi, Mariano. (1998). El ABC de la tarea docente: Currículum y enseñanza. Buenos Aires: Aique.

Lerner, Delia. (2001). Leer y escribir en la escuela: Lo real, lo posible y lo necesario. México: Fondo de Cultura Económica.

Litwin, Edith. (1997). Las configuraciones didácticas: Una nueva agenda para la enseñanza superior. Buenos Aires: Paidós.

Lomas, Carlos. (1999). Cómo enseñar a hacer cosas con las palabras. Barcelona: Paidós.

López, Gladys y Arciniegas, Esperanza. (2004). Metacognición, lectura y construcción de conocimiento. El papel de los sujetos en el aprendizaje significativo. Cali: Universidad del Valle.

Mateos, Mar. (2001). Metacognición y educación. Buenos Aires: Aique.
Mayor, Juan, Suengas, Aurora \& González M., Javier. (1993). Estrategias metacognitivas: Aprender a aprender y aprender a pensar. Madrid: Síntesis.

Monereo, Carles. (1997). El asesoramiento en el ámbito de las estrategias de aprendizaje. En: Monereo, Carles. (coord.). Estrategias de aprendizaje. Madrid: Antonio Machado Libros.

Palincsar, Annemarie y Brown, Anne. (1996). La enseñanza para la lectura autorregulada. En: Resnick, Lauren y Klopfer, Leopold. Currículum y cognición. Buenos Aires: Aique.

Rincón, Gloria et ál. (2003). Entre textos: La comprensión de textos escritos en la educación primaria. Cali: Universidad del Valle.

Santiago G., Álvaro W., Castillo, P., Myriam C. \& Ruiz, R., Jaime. (2005). Lectura, metacognición y evaluación. Bogotá: Alejandría Libros.

Smith, Frank. (1990). Para darle sentido a la lectura. Madrid: Antonio Machado Libros.

Solé, Isabel. (1998). Estrategias de lectura. Barcelona: Grad.

Tardif, Jacques. (1997). La evaluación del saber-leer: un asunto más de competencia que de actuación. En: Rodríguez, Emma y Lager, Elizabeth. (coords.). La lectura. Cali: Universidad del Valle. 